\title{
About influence of gravity on heat conductivity process of the Planets
}

\author{
S.O. Gladkov * \\ Moscow Aviation Institute, National Research University, Volokolamskoe Shosse 4, Moscow, Russia \\ Anil Yadavi \\ Department of Physics, Galgotias College of Engineering and Technology, Greater Noida 201306, India \\ Saibal Ray \\ Department of Physics, Government College of Engineering 83 Ceramic Technology, Kolkata 700010, West Bengal, India \\ F. Rahaman 8 \\ Department of Mathematics, Jadavpur University, Kolkata 700032, West Bengal, India
}

(Dated: today)

\begin{abstract}
In the present study it is shown that the interaction of a quasi-static gravitational wave through density fluctuations gives rise to a heat conductivity coefficient and hence temperature. This fact is a very important characteristics to establish a heat equilibrium process of such massive body as the Earth and other Planets. To carry out this exercise general mechanism has been provided, which makes a bridge between classical physics and quantum theory, and specific dependence of heat conductivity coefficient in wide region is also calculated.
\end{abstract}

PACS numbers: 04.30.-w, 04.30.Nk, 44.10.+i

Problems related to study of heat conduction process in the various bodies an enormous number of attempts are reported in the literature (see, for example, the original paper [1-3] and the monograph [4, 5]). In this case a single distinguishing feature among all of them is a model representation of the structure of the body. If it is a crystalline insulator, then Debye's phonon model does 'work'. On the other hand, for a complex heterogeneous structure, which are in particular porous body, then the Debye's model as well as the gas approximation is applicable. This allows us not only to do analytical description correctly and proper implementation to several experiments [ $[$ ]), but also it predicts number of additional effects [7] (as reflected in [8] and later on confirmed by experiments $[9,10])$.

In this paper we investigate an influence on the process of heat transfer mechanisms associated with the interaction of gravitation via the density fluctuations. As we shall see later, it is extremely important for any massive body, such as Earth, to account for this type of effect. As an object of present research, we choose the simplest stuff and confine ourselves within the dielectric composition of the matter. To determine the contribution of gravity to the coefficient of thermal conductivity, one should note that if there are multiple mechanisms with different physical nature, then the thermal conductivity becomes an additive function and is determined by the sum of contributions of relevant mechanisms. For dielectrics such contribution will consist of only two components and can be represented as

$$
\kappa=\kappa_{p}+\kappa_{\mathrm{g}},
$$

where $\kappa_{p}$ is the coefficient of thermal conductivity due to density fluctuations (in the Debye's model of phonons) and an additional contribution $\kappa_{\mathrm{g}}$ is the coefficient of thermal conductivity due to the gravity.

If the temperature behavior is understood, then its dependence can be determined by the formula

$$
\kappa_{p}=\left\{\begin{array}{c}
A T^{3} \text { at } T \ll \bar{\theta}_{D} \\
B \text { at } T \gg \bar{\theta}_{D}
\end{array}\right.
$$

Here $A$ and $B$ are well-defined coefficients (for more details one can look at the Ref. 11] or the classical monographs [12, 13] ), and $\bar{\theta}_{D}$ is the average energy of thermal vibrations (for crystalline body this is simply the Debye temperature).

To calculate the coefficient of gas we use the simplest approximation and represent it in the form

$$
\kappa_{\mathrm{g}}=\frac{1}{3} C_{\mathrm{g}} c^{2} \tau_{\mathrm{g}-p},
$$

where $c$ is the speed of light, $C_{\mathrm{g}}$ is the heat capacity of gravity waves per unit volume of the body and $\tau_{\mathrm{g}-p}$ is the relaxation time of the transfer of energy between the gravitational wave and phonons.

Most effective mechanism of phonon interaction with the gravitational wave is the process of disintegration of the gravitational wave into two phonons. This act of interaction is characterized by the following two conservation laws:

(i) The law of conservation of energy

$$
\hbar \omega_{\mathrm{g}}(k)=\hbar\left[\omega_{p}\left(q_{1}\right)+\omega_{p}\left(q_{2}\right)\right],
$$

ii) The law of conservation of momentum

$$
\vec{k}=\vec{q}_{1}+\vec{q}_{2},
$$


where $\hbar$ is the Planck constant, $\vec{k}$ and $\vec{q}_{1,2}$ are the wave vectors of gravitational waves and phonon wave respectively. The laws of dispersion (frequency dependence of the wave vector) are defined as $\omega_{\mathrm{g}}(k)=c k$ and $\omega_{p}(q)=c_{s} q$, where $c_{s}$ is the average speed of sound for the Earth.

The simplest algebraic analysis of conservation laws (4) - (5) leads to the conclusion that the wave vector of the virtual phonon must be enclosed in a narrow range, such that

$$
\frac{k}{2}\left(\frac{c}{c_{s}}-1\right) \leq q_{1} \leq \frac{k}{2}\left(\frac{c}{c_{s}}+1\right) .
$$

Since the speed of light is much greater than the speed of sound, then $(6)$ reduces to $q_{1} \approx q_{2} \approx k c / 2 c_{s}$. This is the energy of the gravitational wave which is approximately equally distributed between the two virtual phonons.

To write down properly the expression for amplitude of the static gravitational field, we must remember that the gravitational potential for distances $r \geq R$, where $R$ is radius of the body, can be used in the following form [14]:

$$
\varphi=\frac{r_{\mathrm{g}} c^{2}}{2 r}
$$

where $r_{\mathrm{g}}$ is the gravitational radius of the planet under consideration, as defined by $r_{\mathrm{g}}=2 G M / c^{2}$, here $G$ and $M$ are the universal constant of gravitation and mass of planet respectively.

The transition from classical expression (7) to quantum representation is now a fundamental requirement which can be obtained through the Navigation Rules [15]. Then the second quantization formalism in the quasi-static approximation can be written as

$$
\begin{array}{r}
\hat{\varphi}(\vec{r}, t)=i \sum_{k} \sqrt{\frac{2 \pi G \hbar c}{V_{\mathrm{g}} k}} \times \\
{\left[\hat{c}_{k}^{+} e^{-i\left(\vec{k} \cdot \vec{r}-\omega_{\mathrm{g}} t\right)}+\hat{c_{k}} e^{i\left(\vec{k} \cdot \vec{r}-\omega_{\mathrm{g}} t\right)}\right],}
\end{array}
$$

where $\hat{c}_{k}\left(\hat{c}_{k}^{+}\right)$is the annihilation operator (creation) of gravitational waves and $V_{\mathrm{g}}=4 \pi r_{\mathrm{g}}^{3} / 3$ is the quantization volume of corresponding gravitational field.

To get the Hamiltonian of the interaction between density fluctuations and gravitational waves, we shall now employ some basic postulates of differential geometry. Indeed, in a curved space the volume integral can be represented in the following invariant form:

$$
J=\int_{V}(\ldots) \sqrt{-g} d V
$$

where $g$ is determinant of the metric tensor. Here 'minus' sign characterizes the pseudo-Euclidean space-time. In almost static gravitational field the time component of the metric tensor is given by 14

$$
g_{00} \approx 1-\frac{2 \varphi}{c^{2}} \text {. }
$$

The above indices run upto four values and the index corresponds to the time coordinate. Actually here the situation demands that $\sqrt{-g}=\sqrt{-\left(g_{0}+\delta g\right)} \approx$ $\sqrt{-g_{0}}\left(1+\frac{\delta g}{2 g_{0}}\right)$, where $\delta g \ll g_{0}$, as in a flat pseudoEuclidean spacetime we get here the similar result.

This means that the transition to the non-relativistic approximation of interaction with an almost static gravitational field must be described by an expression in operator form. Thus the desired interaction of gravitational waves with the density fluctuations, whose role in the formal language of phonons is played by the strain tensor, is given by

$$
\hat{H}_{i n t}=\frac{\bar{\theta}_{D}}{V c^{2}} \int_{V} \hat{u}_{\alpha \beta}^{2} \hat{\varphi} d V
$$

where $a$ is the average distance between nodes of localized oscillating structures on the substance and is related to $\bar{\theta}_{D}$ as follows: $\bar{\theta}_{D}=\hbar \bar{c}_{s} / \bar{a}$.

The strain tensor $u_{\alpha \beta}$ associated with the displacement vector points of the continuum ratio $u_{\alpha}$ can be written by

$$
u_{\alpha \beta}=\frac{1}{2}\left(\frac{\partial u_{\alpha}}{\partial x_{\beta}}+\frac{\partial u_{\beta}}{\partial x_{\alpha}}\right) .
$$

Again, secondary-quantized expression for the displacement vector has the following form

$$
\begin{array}{r}
\hat{u}_{\alpha}=\sum_{q, e} e_{\alpha}\left(\frac{\hbar}{2 \rho V \omega_{p}(q)}\right)^{\frac{1}{2}} \times \\
{\left[\hat{b}_{q}^{+} e^{-i\left(\vec{q} \cdot \vec{r}-\omega_{p} t\right)}+\hat{b}_{q} e^{i\left(\vec{q} \cdot \vec{r}-\omega_{p} t\right)}\right],}
\end{array}
$$

where $\rho$ is the density structure, $V$ is its volume, whereas $\hat{b}_{q}^{+}\left(\hat{b}_{k}\right)$ is the creation (annihilation) of a phonon with wave vector $\vec{q}$ and $\vec{e}_{\alpha}$ is the polarization vector of the sound wave.

Now if we substitute above Eq. (13) into Eq. (12), and it in turn to the Hamiltonian (11), then considering Eq. (9) we find the expected expression for interaction of energy density fluctuations with the gravitational field as follows:

$$
\begin{array}{r}
\hat{H}_{i n t}=\frac{\pi \bar{\theta}_{D} \hbar}{2 V \rho c_{s} c^{2}} \sqrt{\frac{2 \pi G c \hbar}{V_{\mathrm{g}}}} \times \\
\sum_{k, q_{1}, q_{2}} \frac{\left(\vec{e}_{1} \cdot \vec{q}_{1}\right)\left(\vec{e}_{2} \cdot \vec{q}_{2}\right)}{\sqrt{k q_{1} q_{2}}}\left[\hat{b}_{q_{1}}^{+}-\hat{b}_{-q_{1}}\right]\left[\hat{b}_{q_{2}}^{+}-\hat{b}_{-q_{2}}\right] \times \\
{\left[\hat{c}_{k}^{+}+\hat{c}_{-k}\right] \Delta\left[\vec{k}+\overrightarrow{q_{1}}+\overrightarrow{q_{2}}\right](1}
\end{array}
$$


where $\Delta$ is function of $x$ such that $\Delta(x)=1$ if $x=0$ and $\Delta(x)=0$ for all $x \neq 0$.

Knowing the interaction (14) it is now easy to find out an expression for relaxation time $\tau_{\mathrm{g}-p}$ of the gravitational wave appearing in the formula (3). In fact, if we use the recipe described in detail in Ref. [15], after some simple calculations taken into account, the conservation laws (4) and (5) through (6) will provide the desired decay time of the gravitational wave into two phonons:

$$
\frac{1}{\tau_{\mathrm{g}-p}(k)} \approx \frac{\pi^{2}}{32}\left(\frac{V}{V_{g}}\right) \frac{G \bar{\theta}_{D}^{2}}{\hbar c_{s}^{5}}\left(\frac{\hbar}{\rho c_{s} \bar{a}^{4}}\right)^{2}(\bar{a} k)^{2} k c\left(1+2 \bar{N}_{k}\right),
$$

where $\bar{N}_{k}=\frac{1}{e^{\hbar c_{s} k / T}-1}$ is the equilibrium phonon distribution function of Bose-Einstein statistics in standard form. The Boltzmann constant here after to be equal to unity. Note that in (15) there was a big factor $V / V_{\mathrm{g}}$ due to transitions from summation to integration over the region of phase space phonon. It can be shown in the form

$$
\sum_{q} f(q)=V \int_{V} f(q) \frac{d^{3} q}{(2 \pi)^{3}}
$$

For averaging formula (15) on the distribution of Planck statistics, which are subject to gravitational waves, we use the following simple rule:

$$
\overline{(\ldots)}=\frac{\int_{0}^{\infty} k^{2} \bar{f}_{k}(\ldots) d k}{\int_{0}^{\infty} k^{2} \bar{f}_{k} d k}
$$

where $\bar{f}_{k}=\left(e^{\hbar c k / T}-1\right)^{-1}$.

The above rule leads us to the following expression for the average relaxation time of the gravitational wave

$$
\frac{1}{\bar{\tau}_{\mathrm{g}-p}} \approx \frac{\pi^{2}}{32}\left(\frac{V}{V_{\mathrm{g}}}\right) \frac{G \bar{\theta}_{D}^{2} c}{\hbar c_{s}^{5} \bar{a}}\left(\frac{\hbar}{\rho c_{s} \bar{a}^{4}}\right)^{2}\left(\frac{\bar{a} T}{\hbar c}\right)^{3}(1+2 \bar{N}(u)),
$$

where $u=c_{s} / c$ and for the constraint $u \ll 1$, we get $\bar{N}(u) \approx 1 / u=c / c_{s}$.

Therefore, under the above approximation for a spherical body one can assume the equation (16) in its modified form

$$
\frac{1}{\bar{\tau}_{\mathrm{g}-p}} \approx \frac{\pi^{2}}{16} \frac{G \bar{\theta}_{D}^{2} c^{2}}{\hbar c_{s}^{6} \bar{a}}\left(\frac{\hbar}{\rho c_{s} \bar{a}^{4}}\right)^{2}\left(\frac{\bar{a} T}{\hbar c}\right)^{3}\left(\frac{R}{r_{\mathrm{g}}}\right)^{3} .
$$

Since the heat capacity of the particles with a linear range of the wave vector (as well as in the Debye model) is proportional to $T^{3}$, then according to Eqs. (17) and (3) we have

$$
\kappa_{\mathrm{g}}=\frac{1}{3} C_{\mathrm{g}} c^{2} \tau_{\mathrm{g}-p} \sim\left(\frac{T}{\hbar c}\right)^{3} c^{2} \bar{\tau}_{\mathrm{g}-p}=\text { constant }=D,
$$

where the new coefficient $D$ is defined as

$$
D \approx\left(\frac{c_{s}}{c}\right)^{3}\left(\frac{\rho c_{s} \bar{a}^{4}}{\hbar}\right)^{2} \frac{\hbar c_{s}^{6}}{G \bar{a}^{2} \bar{\theta}_{D}^{2}}\left(\frac{r_{\mathrm{g}}}{R}\right)^{3} .
$$

Therefore, by summing Eqs. (2) and (18), we find the expression for thermal conductivity for massive structure as follows

$$
\kappa=\left\{\begin{array}{c}
A T^{3} \text { if } T \ll \bar{\theta}_{D} \\
B \text { if } T \gg \bar{\theta}_{D}
\end{array}+D\right.
$$

At this point it would be very appropriate to give an estimate of average relaxation time of the quasi-static gravitational wave. Hence, according to Eq. (17), we obtain the order of magnitude for relaxation time as

$$
\bar{\tau}_{\mathrm{g}-p} \approx 10^{-8} c .
$$

For the above estimation we have used the following numerical values of the parameters: $\hbar=10^{-27} \mathrm{erg}-\mathrm{s}, G=$ $6.67 \times 10^{-8} C G S, \bar{\theta}_{D}=100 \mathrm{~K}, c=3 \times 10^{10} \mathrm{~cm}-$ $\mathrm{s}^{-1}, \quad c_{s}=10^{5} \mathrm{~cm}-\mathrm{s}^{-1}, \rho=5.5 \mathrm{~g}-\mathrm{cm}^{-3}, \bar{a}=$ $10^{-8} \mathrm{~cm}, R=6.4 \times 10^{8} \mathrm{~cm}, r_{\mathrm{g}}=10 \mathrm{~cm}, T=300 \mathrm{~K}=$ $3 \times 10^{-14} \mathrm{erg}$.

Note that above value of relaxation time represents a very fast thermalization process of gravitational wave and points to the need for a massive body.

To summarize, in the present paper we have studied influence of gravity in the thermal conductivity process of massive bodies. Actually the interaction of a quasistatic gravitational wave via density fluctuations gives rise to a heat conductivity coefficient $\kappa$ in connection to a temperature $T$. Therefore, it is important to establish a heat equilibrium process of such massive body like the Earth and other Planets.

As a major result of the investigation we would like to highlight the following two important points:

(1) In the description of thermal conductivity of massive bodies it is extremely important to consider the interaction of gravitational waves with the remaining substance, however its composition will be determined by the particular material of the structure.

(2) The coefficient of thermal conductivity of such bodies due to gravitational waves increases with temperature which eventually gets saturated and tends to a constant value such that at high temperature it ceases to depend on any external influence.

SR and FR are thankful to the authority of InterUniversity Centre for Astronomy and Astrophysics, Pune, India for providing Visiting Associateship under which a part of this work was carried out.

\footnotetext{
* Electronic address: sglad@newmail.ru
} 
$\dagger$ Electronic address: abanilyadav@yahoo.co.in

¥ Electronic address: saibal@iucaa.ernet.in

$\S$ Electronic address: rahaman@iucaa.ernet.in

[1] A.I. Akhiezer, V.G. Bar'yakhtar, and M.I. Kaganov, Progress of the Physical Sciences 12, 3 (1960).

[2] D.N. Zubarev, Progress of the Physical Sciences 71, 71 (1960).

[3] S.O. Gladkov, Solid State Communications 82, 919 (1992); Ibid 94, 787 (1995).

[4] H.S. Carslaw and J. Jaeger, Conduction of Heat in Solids Oxford, 487 (1960).

[5] A. Misnar, Thermal Conductivity of Solids, Liquids, Gases and their Compositions, Wiley 345 (1968).

[6] S.O. Gladkov, Physics of Metals and Metallography 94, 30 (2002).

[7] S.O. Gladkov, Physica B - Condensed Matter 167B, 159 (1990).

[8] S.O. Gladkov, Dielectric Properties of Porous Media,
Springer, 261 (2003)

[9] R.Y. Abraytis, A.K. Dargis, A.A. Rusyatskas, and E.J. Sakalauskas, Refractories and Technical Ceramics , 22 (1999); Ibid , 33 (2000); Ibid , 48 (2000).

[10] N.N. Grishin, O.A. Belogurova, and A.G. Ivanov, Refractories and Industrial Ceramics , 45 (2002).

[11] S.O. Gladkov and I.V.Gladyshev, Physics of Status Solids 47, 1143 (2004).

[12] I.M. Lifshitz, M. Ya. Azbel, and M.I. Kaganov, Electron Theory of Metals, Moscow: Science, 415 (1971).

[13] E.M. Lifshitz and L.P. Pitaevskii, Physical Kinetics, Moscow: Science, 527 (1979)

[14] L.D. Landau and E.M. Lifshitz, Field Theory, Vol 2, Moscow: Science 504 (1973)

[15] S.O. Gladkov, Physics of Composites: Thermodynamic and Dissipative Properties, Moscow: Science, 330 (1999) 